\title{
Influence of the waist diameters on transmission characteristics and strain sensitivity of microtapered long-period fiber gratings
}

\author{
Min-Seok Yoon, ${ }^{1}$ Hyun-Joo Kim, ${ }^{1}$ Sung-Jae Kim, ${ }^{1}$ and Young-Geun Han ${ }^{1,2, *}$ \\ ${ }^{1}$ Department of Physics, Hanyang University, Seoul 133-791, South Korea \\ ${ }^{2}$ California Institute of Technology (Caltech), 1200 E. California Blvd., Pasadena, California 91125, USA \\ ${ }^{*}$ Corresponding author: yghan@hanyang.ac.kr
}

Received May 16, 2013; revised June 24, 2013; accepted June 26, 2013;

posted June 27, 2013 (Doc. ID 189391); published July 22, 2013

\begin{abstract}
Transmission characteristics of microtapered long-period fiber gratings (MTLPGs) and their strain and temperature sensitivities with variations in the waist diameters are investigated theoretically and experimentally. Transmission characteristics of MTLPGs strongly depend on the waist diameter of the tapered optical fiber (TOF) because of the modification of the effective index difference between the core and the cladding modes. Based on the photoelastic effect, the resonant wavelengths of MTLPGs with variations in strain shift to shorter wavelengths. The strain sensitivity of the MTLPG with a waist diameter of $25 \mu \mathrm{m}$ is improved by a factor of 20 compared with that of a $125 \mu \mathrm{m}$ long-period fiber grating. The temperature sensitivities of MTLPGs are also enhanced by reducing the waist diameter of the TOF. (C) 2013 Optical Society of America

OCIS codes: (060.2310) Fiber optics; (060.2370) Fiber optics sensors; (230.3990) Micro-optical devices; (050.2770) Gratings.

http://dx.doi.org/10.1364/OL.38.002669
\end{abstract}

High sensitivities of long-period fiber gratings (LPGs) to external perturbations, such as temperature, strain, and bending, have led to much interest in a variety of fiberoptic sensors [1-5]. LPGs with a large scale periodic axial perturbation in a single-mode fiber (SMF) can couple a forward propagating core mode $\left(\mathrm{HE}_{11}\right)$ to the forward propagating cladding modes $\left(\mathrm{HE}_{1 m}\right)$ in a SMF, which results in resonant peaks in the transmission. The large period of LPGs, however, makes them insensitive to variations in strain. Recently LPGs inscribed in the microfibers have been proposed to improve their performance as fiber-optic sensors [3-5] . Since microfibers have high fractional evanescent fields in air, strong evanescent field coupling in microfiber-based LPGs is capable of enhancing their sensitivity to ambient index change $[4,5]$. Since microfibers are usually fabricated by tapering a conventional SMF [3-5], it is necessary to investigate the effect of waist diameter of tapered optical fiber (TOF) on the transmission characteristics, strain, and temperature sensitivities of the LPG. In this Letter, the effect of waist diameter of TOF on the transmission characteristics, strain, and temperature sensitivities of microtapered long-period fiber gratings (MTLPGs) is investigated. Since the variation of the waist diameter of TOF changes the refractive index difference between the core and cladding modes, various cladding modes can be coupled from the fundamental core mode in MTLPGs with distinct waist diameters. The increase of strain makes the resonant wavelengths of the MTLPGs shift to shorter wavelengths because of the photoelastic (PE) effect. Decreasing the waist diameter improves the strain sensitivity of the MTLPG to be 20 times higher than that of a $125 \mu \mathrm{m} \mathrm{LPG} \mathrm{[1].} \mathrm{Since}$ MTLPGs based on conventional SMF have positive thermal properties, the resonant wavelengths should be shifted to longer wavelengths as temperature increases. The temperature sensitivities of MTLPGs can be somewhat increased by reducing the waist diameters because of the excitation of different cladding modes in the MTLPGs with various waist diameters.

Figure 1 shows the fabrication procedure and microscopic images of MTLPGs with various waist diameters $(D)$. After manufacturing various TOFs with waist diameters $(D)$ of $125,100,75,50$, and $25 \mu \mathrm{m}$ by tapering conventional SMFs with a flame brushing technique []ㅡ, we periodically pulled and elongated them. The periodic formation of the locally tapered diameter $(d)$ changes the effective refractive index, resulting in the realization of various MTLPGs. The values of $d$ in comparison with the waist diameter $(D)$ should be further reduced and were measured to be $\sim 70,60,50,40$, and $20 \mu \mathrm{m}$, as seen in Fig. 2. The grating periods $(\Lambda)$ of MTLPGs with waist diameters $(D)$ of $125,100,75$, and $50 \mu \mathrm{m}$ were the same, at $\sim 1.2 \pm 0.1 \mathrm{~mm}$.

Figure 2(a) shows theoretical results for field distributions of the core and cladding modes as the fundamental core mode is propagating through MTLPGs with different waist diameters $(D)$. The field distribution of the fundamental core mode is changed as the waist diameters $(D)$ are reduced. The normalized intensity profiles of core modes with variations in waist diameters $(D)$ are shown in Fig. 2(b). Since the mode field diameter (MFD) of the

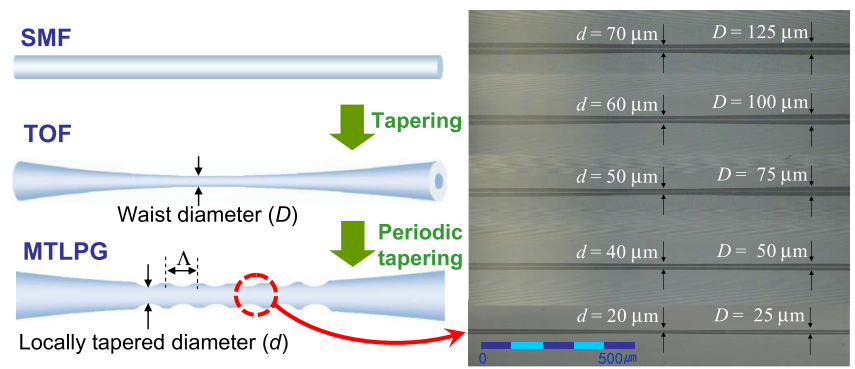

Fig. 1. Scheme for the MTLPGs inscribed on different TOFs. Microscopic images of the MTLPGs with various waist diameters are shown. 
(a)

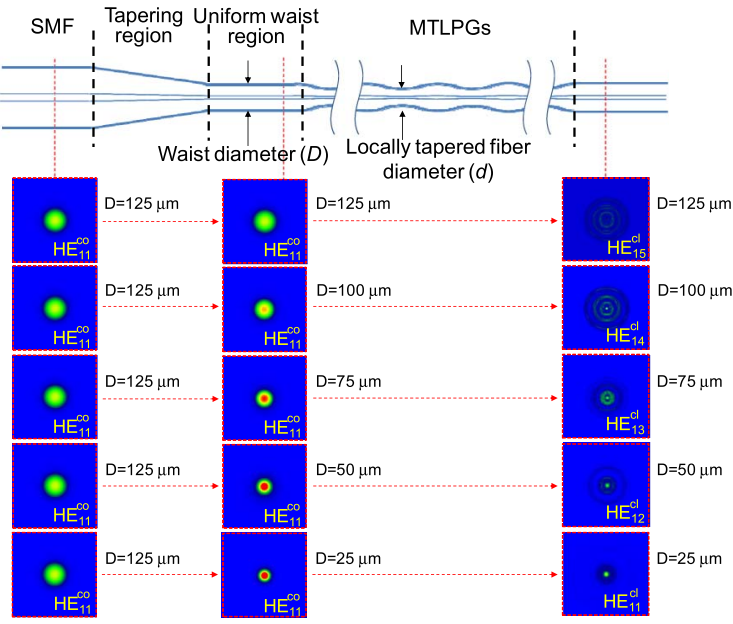

(b)

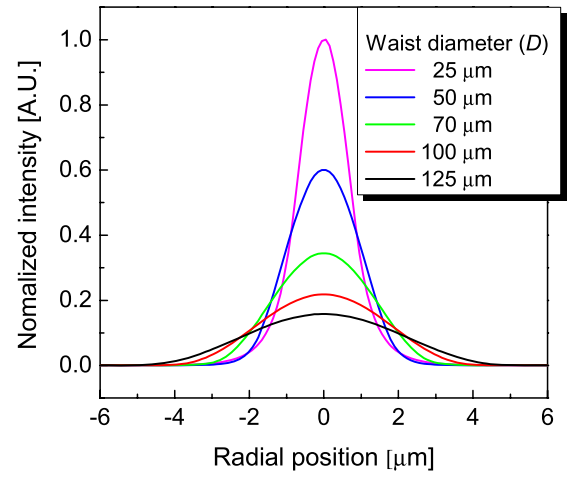

Fig. 2. (a) Theoretical results for field distributions of the core and cladding modes in MTLPGs with different waist diameters of $125,100,75,50$, and $25 \mu \mathrm{m}$ and (b) theoretical results for the normalized intensity profiles of core modes with variations in waist diameter.

fundamental core mode in the TOF is proportional to the waist diameter, the MFD should be reduced, the field confinement of the core mode should be enhanced, and the waist diameter $(D)$ decreases.

Figure 3(a) shows experimental results for transmission spectra of the MTLPGs with various waist diameters (D) of $125,100,75,50$, and $25 \mu \mathrm{m}$. The measured mode field images of the cladding modes $\left(\mathrm{HE}_{1 \mathrm{~m}}\right)$ coupled from the fundamental core mode $\left(\mathrm{HE}_{11}\right)$ in the MTLPGs are shown in the insets. Mode coupling between the core and cladding modes occurs as the core mode propagates along the MTLPGs because the core and cladding regions of the TOFs are locally and periodically reduced again, which results in the periodic variation of the effective refractive indices. Therefore, resonant wavelengths resulting from mode coupling should be exhibited in the transmission spectra of the MTLPGs. The different cladding modes in four types of MTLPGs with distinct waist diameters $(D)$ were excited from the fundamental core mode. Figure 3(b) shows theoretical results for the effective indices of the core and cladding modes as functions of different waist diameters $(D)$. The variation of waist diameter may change the field distribution and, consequently, the effective indices of the core and cladding modes. Since the $V$-parameter is generally proportional to the physical diameters of the optical fibers, the reduction in waist diameter decreases the effective indices of the core and cladding modes, as seen in Fig. 3(b) (a)
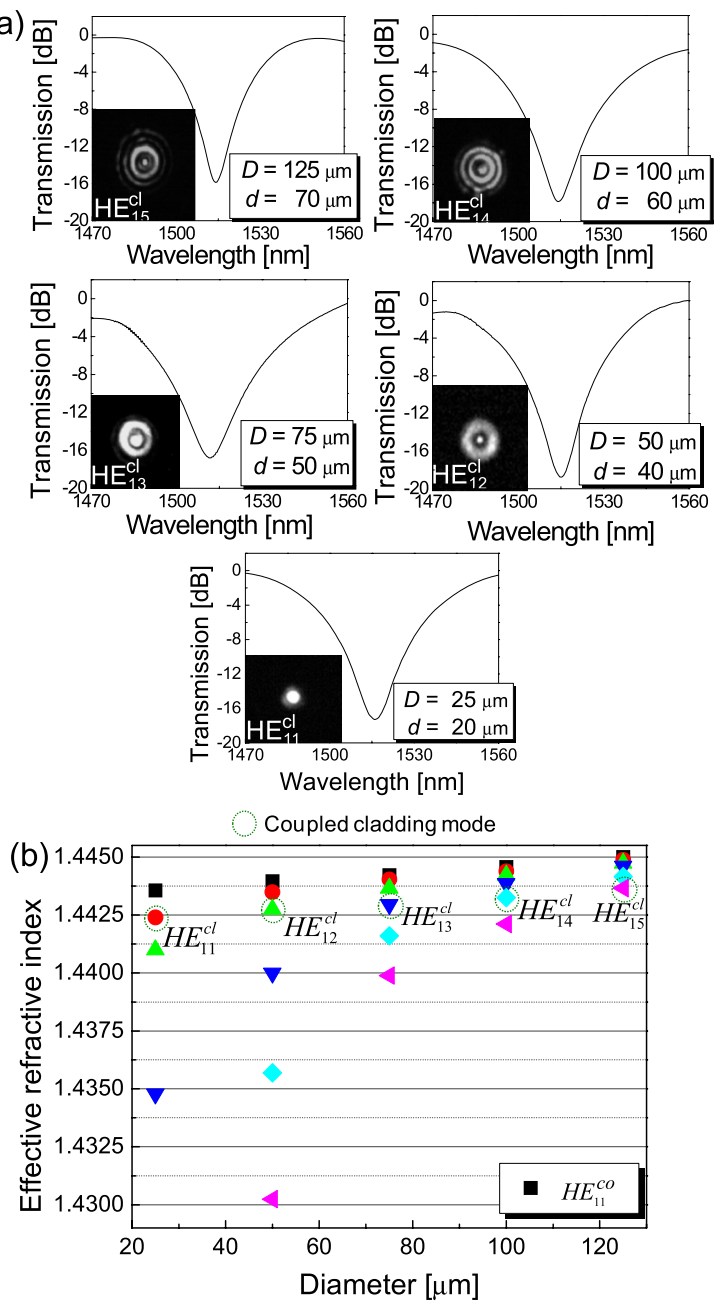

Fig. 3. (a) Experimental results for transmission spectra of the MTLPGs with various waist diameters. The measured mode field images of the cladding modes $\left(\mathrm{HE}_{1 \mathrm{~m}}\right)$ coupled from fundamental core mode $\left(\mathrm{HE}_{11}\right)$ are shown in the insets. (b) Theoretical results for effective indices of the core and cladding modes with variations in waist diameter.

[6]. However, the reduction in the effective index of the cladding mode is much higher than that in the core mode and it is proportional to the cladding mode order $(m)$ because of different field distribution with variations in waist diameter $(D)$ [7]. Therefore, the difference of effective indices between the core and cladding modes is changed depending on the distinct waist diameter. This means that MTLPGs with different waist diameters $(D)$ should couple the fundamental core mode to different cladding modes. As the waist diameter $(D)$ decreases, the low-order cladding mode must be excited from the fundamental core mode in the MTLPG. As seen in Figs. 2(a) and 3(a), the experimental results are in good agreement with the theoretical results.

According to the PE effect, externally applied strain reduces the effective index difference between the core and cladding modes, which results in a resonant wavelength shift to shorter wavelengths [8]. The variation of the refractive indices in the tapered $\left(\delta n_{D}(r)\right)$ and the locally tapered regions $\left(\delta n_{d}(r)\right)$ based on the PE effect after applying strain $(\varepsilon)$ can be expressed by $[\underline{9}, \underline{10}]$ 


$$
\begin{aligned}
\delta n_{D, d}(r) & =-\frac{1}{2} p\left[n_{D, d}(r)\right]^{3} \varepsilon_{D, d} \\
\varepsilon_{D} & =\frac{4 F}{Y \pi D^{2}} \\
\varepsilon_{d} & =\frac{4 F}{Y \pi d^{2}}
\end{aligned}
$$

where $n_{D}(r)$ and $n_{d}(r)$ are the effective refractive indices in the tapered and the locally tapered regions before applying strain to the MTLPGs. $p$ and $Y$ are a PE constant and Young's modulus, respectively. $F$ is the applied force. $D$ is the waist diameter of the TOF, and $d$ is the diameter of the locally tapered region in the MTLPG $(D>d)$. Assuming that $n_{D}(r)$ is similar to $n_{d}(r)$ without the applied strain, the strain-induced refractive index change based on the PE effect $\left(\Delta n_{\text {strain }}(r)\right)$ can be derived as [10]

$$
\Delta n_{\text {strain }}(r)=\delta n_{d}(r)-\delta n_{D}(r)=-\frac{1}{2} p\left[n\left(r_{D}\right)\right]^{3}\left[\frac{D^{2}}{d^{2}}-1\right] \varepsilon_{D}
$$

When strain is applied to the MTLPG, $\delta n_{D}(r)$ is smaller than $\delta n_{d}$ because of the smaller value of $d$ than $D$. This means that $\Delta n_{\text {strain }}(r)$ is negative $\left(\Delta n_{\text {strain }}(r)<0\right)$ because $\varepsilon_{D}$ and $\varepsilon_{d}$ are inversely proportional to two diameters, such as $D$ and $d$. By considering the phasematching condition in the MTLPGs and Eq. (1), the strain sensitivity of the resonant wavelength $\left(d \lambda_{P} / d \varepsilon\right)$ can be derived as

$$
\begin{aligned}
\frac{\partial \lambda_{P}}{\partial \varepsilon}= & -\underbrace{\frac{2 F}{Y \pi}\left(\frac{1}{d^{2}}-\frac{1}{D^{2}}\right)\left(p^{c o}\left[n_{c o, D}(r)\right]^{3}-p^{c l}\left[n_{c l, D}^{(m)}(r)\right]^{3}\right)}_{\mathrm{PE}} \cdot \Lambda \\
& +\underbrace{\frac{\partial \lambda_{p}}{\partial \Lambda} \frac{\partial \Lambda}{\partial \varepsilon}}_{\mathrm{WD}},
\end{aligned}
$$

where $p^{\mathrm{co}}$ and $p^{\mathrm{cl}}$ are PE constants of the core and cladding regions, respectively. $n_{\mathrm{co}, D}(r)$ and $n_{\mathrm{cl}, D}^{(m)}(r)$ are effective refractive indices of the core and cladding modes as a function of radius without the applied strain. $\Lambda$ is the grating period. The strain sensitivity is determined by the $\mathrm{PE}$ and the waveguide dispersion (WD) terms in Eq. (3). As seen in this equation, the MTLPG with a large period has negative strain sensitivity because of the negative contribution of the PE factor, which is higher than the WD term. The strain sensitivity of the MTLPG should be improved by diminishing $d$ and $D$ together because the strain sensitivity is inversely proportional to the squares of two diameters, such as $d^{2}$ and $D^{2}$. On the other hand, the increase of temperature makes the resonant wavelength of the MTLPG shift to longer wavelengths because of the positive thermal expansion and thermo-optic coefficients of the TOF. The temperature sensitivity of the MTLPGs can be expressed by [11]

$$
\begin{aligned}
\frac{\partial \lambda_{p}}{\partial T}= & \lambda_{p} \cdot \frac{\frac{\partial \lambda_{p}}{\partial \Lambda}}{n_{\mathrm{co}, D}(r)-n_{\mathrm{cl}, D}^{(m)}(r)} \\
& \cdot\left(\alpha+\frac{\xi_{\mathrm{co}} n_{\mathrm{co}, D}(r)-\xi_{\mathrm{cl}} n_{\mathrm{cl}, D}^{(m)}(r)}{n_{\mathrm{co}, D}(r)-n_{\mathrm{cl}, D}^{(m)}(r)}\right),
\end{aligned}
$$

where $\alpha$ is the thermal expansion coefficient $(>0) \cdot \xi_{\text {co }}$ and $\xi_{\mathrm{cl}}$ are the thermo-optic coefficients in the core and cladding materials, respectively. The positive thermo-optic and thermal expansion coefficients of a conventional SMF with a germanium-doped core makes the resonant wavelength of the MTLPG shift to longer wavelengths as temperature increases.

Figures 4(a) and 4(b) show experimental and theoretical results for resonant wavelength shifts of the MTLPGs as functions of strain and waist diameter, respectively. For the theoretical analysis, the $\mathrm{PE}$ constant $(p)$ was $\sim 0.22$ [12]. The resonant wavelengths of the MTLPGs with variations in strain shifted to shorter wavelengths because the PE effect reduces the effective refractive index of the TOF. The strain sensitivities of the MTLPGs with different waist diameters $(D)$ of $125,100,75,50$, and $25 \mu \mathrm{m}$ were measured to be $-0.53,-0.64,-1.20$, -3.05 , and $-10.28 \mathrm{~nm} / \mathrm{m} \varepsilon$, respectively. Because the strain sensitivity of the MTLPGs is inversely proportional to the waist diameter $(D)$, the strain sensitivity of the MTLPGs should be improved by reducing the waist
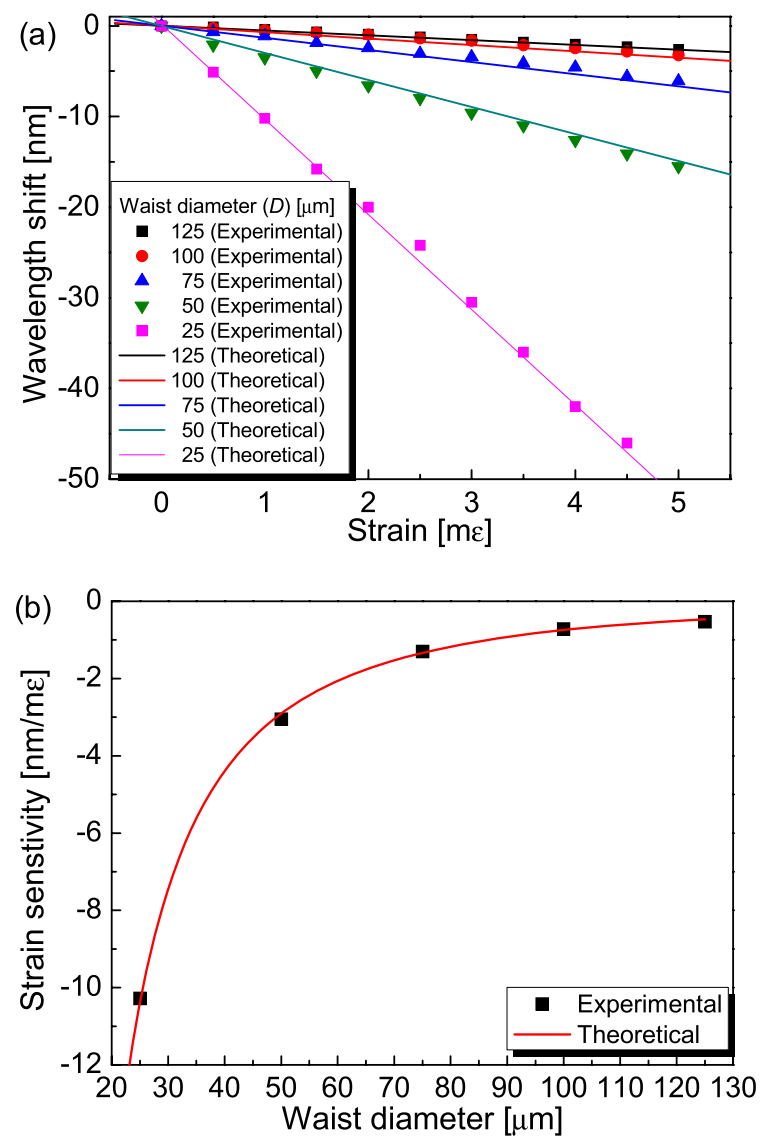

Fig. 4. (a) Experimental (symbols) and theoretical (red line) results for resonant wavelength shifts of MTLPGs as functions of strain and (b) strain sensitivities of MTLPGs as a function of the waist diameter. 
Table 1. Strain Sensitivities of the Fiber Gratings

\begin{tabular}{lc}
\hline Types of Grating & Sensitivity \\
\hline Silica-fiber-based FBG $[13]$ & $1 \mathrm{pm} / \mu \varepsilon$ \\
mPOF-based FBG $[14]^{a}$ & $1.3 \mathrm{pm} / \mu \varepsilon$ \\
Silica-fiber-based L ${ }^{\mathrm{PG}}[\underline{1}]$ & $-0.45 \mathrm{~nm} / \mathrm{m} \varepsilon$ \\
$25 \mu \mathrm{m}$ MTLPG & $-10.28 \mathrm{~nm} / \mathrm{m} \varepsilon$ \\
\hline
\end{tabular}

mPOF: microstructured polymer optical fiber.

diameter. The strain sensitivity of the MTLPG with a waist diameter of $25 \mu \mathrm{m}$ is almost 20 times higher than that of the LPG with a waist diameter of $125 \mu \mathrm{m}$. After calculating the effective indices of the core and cladding modes of the TOFs with variations in waist diameter $(D)$, we theoretically analyzed the strain sensitivities of the MTLPGs with different waist diameters by using Eq. (3), as shown in Fig. 4(b). The theoretical results were in good agreement with the experimental ones. Table 1 shows the comparison of the strain sensitivities for various fiber gratings, including fiber Bragg gratings (FBGs) and LPGs. The strain sensitivity of FBGs is usually higher than that of the LPG because of the small period of the FBGs [13]. In addition, the polymer-fiber-based FBG has higher strain sensitivity than the silica-based FBG because of the high flexibility of the polymer material $[14,15]$. Since decreasing the waist diameter of the optical fiber can further improve the strain sensitivity of the LPG, the proposed MTLPG with high strain sensitivity is applicable to the fiber-optic accelerometer.

Figure $\underline{5}$ shows theoretical and experimental results for resonant wavelength shifts of MTLPGs as functions of temperature. Increasing temperature makes the resonant wavelengths of the MTLPGs shift to longer wavelengths because of positive thermal optic and expansion coefficients of the TOFs based on the SMFs. The temperature sensitivities of the MTLPGs with various waist diameters were measured to be 51.3, 58.5, 59.1, 65.1, and $68.5 \mathrm{pm} /{ }^{\circ} \mathrm{C}$, respectively. The temperature sensitivity of the MTLPG was increased by reducing the waist diameter $(D)$ because it strongly depends on the thermal expansion and optic coefficients and WD with respect to the order of cladding modes [3,11].

In conclusion, the effects of waist diameter of TOFs on the transmission characteristics, strain, and temperature sensitivities of MTLPGs were theoretically and experimentally investigated. In the transmission characteristics of the MTLPGs with various waist diameters, distinct cladding modes were coupled from the fundamental core mode because the different waist diameters changed the refractive index difference between the core and cladding modes. When the MTLPGs are inscribed in various TOFs with different waist diameters, the order of the cladding modes should be decreased as the waist diameters are diminished. The strain and temperature sensitivities of MTLPGs corresponding to various waist diameters were theoretically and experimentally discussed. The external strain made the resonant wavelengths of the MTLPGs shift to shorter wavelengths because of the PE effect. The strain sensitivities of the MTLPGs strongly depend on the waist diameters. Since the variation of the effective refractive index based on

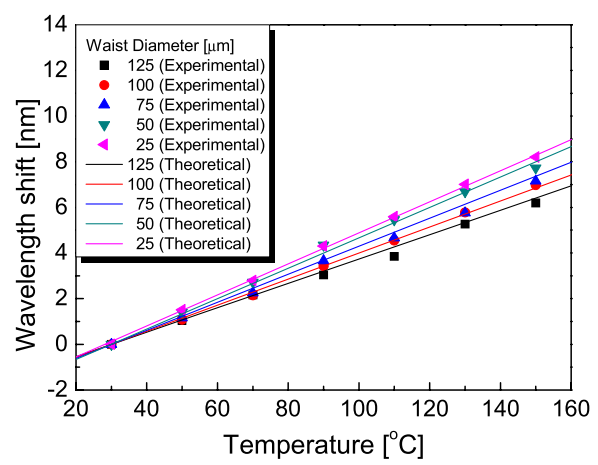

Fig. 5. Experimental (symbols) and theoretical (lines) results for resonant wavelength shifts of MTLPGs with different waist diameters as functions of temperature.

the PE effect is inversely proportional to the waist diameter, the strain sensitivity is significantly improved by reducing the waist diameter. The strain sensitivity of the MTLPG with a waist diameter of $25 \mu \mathrm{m}$ was enhanced to be 20 times higher than that of the $125 \mu \mathrm{m} \mathrm{LPG}$. The applied temperature made the resonant wavelengths of the MTLPGs shift to longer wavelengths because of their positive thermo-optic and expansion properties. The temperature sensitivity was increased by reducing the waist diameters because of the dependence of the thermal properties and the WD of the MTLPGs on the order of the cladding modes.

This research was supported by the Basic Science Research Program through the National Research Foundation of Korea (NRF), funded by the Ministry of Education, Science and Technology (2012R1A1A2000999).

\section{References}

1. Y. J. Rao, Y. P. Wang, Z. L. Ran, and T. Zhu, J. Lightwave Technol. 21, 1320 (2003).

2. H. J. Kim, O. J. Kwon, S. B. Lee, and Y. G. Han, Opt. Lett. 37, 1802 (2012).

3. H. Xuan, W. Jin, and M. Zhang, Opt. Express 17, 21882 (2009).

4. H. Xuan, W. Jin, and S. Liu, Opt. Lett. 35, 85 (2010).

5. L. P. Sun, J. Li, L. Jin, and B. O. Guan, Opt. Express 20 , 18079 (2012).

6. M. S. Yoon, O. J. Kwon, H. J. Kim, S. H. Chu, G. H. Kim, S. B. Lee, and Y. G. Han, J. Korean Phys. Soc. 57, 1747 (2010).

7. J. D. Love, W. M. Henry, W. J. Stewart, R. J. Black, S. Lacroix, and F. Gonthier, IEE Proc. J. Optoelectron. 138, 343 (1991).

8. K. Zhou, H. Liu, and X. Hu, Opt. Commun. 197, 295 (2001).

9. M. S. Yoon, S. Park, and Y. G. Han, J. Lightwave Technol. 30, 1156 (2012).

10. C. Y. Lin and L. A. Wang, J. Lightwave Technol. 19, 1159 (2001).

11. X. Shu and L. Zhang, J. Lightwave Technol. 20, 255 (2002).

12. S. Gupta, T. Mizunami, T. Yamao, and T. Simomura, Appl. Opt. 35, 5202 (1996).

13. H. J. Patrick, G. M. Williams, A. D. Kersey, and J. R. Pedrazzani, IEEE Photon. Technol. Lett. 8, 1223 (1996).

14. A. Stefani, W. Yuan, C. Markos, and O. Bang, IEEE Photon. Technol. Lett. 23, 660 (2011).

15. A. Stefani, S. Andresen, W. Yuan, N. Herholdt-Rasmussen, and O. Bang, IEEE Photon. Technol. Lett. 24, 763 (2012). 